\title{
4'-Metil-3-Fenilakrilofenon Bileşiğinin Moleküler ve Kristal Yapı Analizi
}

\author{
Hasan İNAÇ*1, Nermin KAHVECİ YAĞCI² \\ ${ }^{1}$ Kırıkkale Üniversitesi, Eğitim Fakültesi, Matematik ve Fen Bilimleri Eğitimi Bölümü, Kırıkkale \\ ${ }^{2}$ Kırıkkale Üniversitesi, Fen Edebiyat Fakültesi, Fizik Bölümü, Kırıkkale
}

\section{$\ddot{O} \mathbf{z}$}

Bileşiğin kristal yapısı $\left(\mathrm{C}_{16} \mathrm{H}_{14} \mathrm{O}\right) 296 \mathrm{~K}$ 'de tayin edildi. Tek kristal bileşiğin yapısı tek kristal X-ışını kırınımı yöntemi kullanılarak belirlendi. Kristal yapının birim hücre parametreleri a=14.9921(9) $\AA$, b=9.8460(4) $\AA$, $\mathrm{c}=17.5570(11) \AA, \alpha=90^{\circ}, \beta=105.721(5)^{\circ}, \gamma=90^{\circ}$ ve $Z=8^{\prime}$ dir. Bileşik monoklinik yapıda kristalleşmiş olup uzay grubu C 2/c'dir. Kristal yapıda moleküller arası C-H... O hidrojen bağları, (Bu C-H... O hidrojen bağları zayıf etkileşimli bağlardır) molekülleri üç boyutlu bir ağa bağlar. Bu zayıf etkileşimler, moleküllerin sentrosimetrik dimerik yapı oluşturmasına neden olur.

Anahtar kelimeler: Metilfenil, Fenilakrilofenon, Kristal Yapı, Sentrosimetrik Dimer.

\section{Molecular and Crystal Structure Analysis of 4'-Methyl-3- Phenylacrylophenone Compound}

\begin{abstract}
The crystal structure of the compound, $\left(\mathrm{C}_{16} \mathrm{H}_{14} \mathrm{O}\right)$, was determined at $296 \mathrm{~K}$. The structure of the single crystal compound was determined using a single crystal X-ray diffraction method. The unit cell parameters of the crystal structure are $a=14.9921(9) \AA, b=9.8460(4) \AA, c=17.5570(11) \AA, \alpha=90^{\circ}, \beta=105.721(5)^{\circ}, \gamma=90^{\circ}$ and $Z=8$. The compound crystallizes in monoclinic space group $\mathrm{C} 2 / \mathrm{c}$. In the crystal structure, intermolecular C-H...O hydrogen bonds link the molecules into a three-dimensional network. These weak interactions cause the molecules to form a centrosymmetric dimeric structure.
\end{abstract}

Keywords: Methylphenyl, Phenylacrylophenone, Crystal Structure, Centrosymmetric Dimer.

\section{Giriş}

Dünya Sağlık Örgütünün 2014 tarihli bir raporuna göre Tüberküloz en önemli hastalıklardan biridir [1]. Maalesef tüberküloza karşı geliştirilen çok az ilaç vardır [2-3]. Bu nedenle etkili yeni antimikrobik bileşiklerin sentezi son derece önemli hale gelmiştir. Kalsinler, türevleri kolay üretilen kimyasal bileşiklerdir. Kalkonlar ve sentetik türevleri; anti-inflamatuar, antihistaminik, antioksidan, antitüberküloz, antiülser ve antifungal gibi kapsaml farmakolojik özelliklere sahiptir [4].

Bu çalışmada, bir kalkon türevi ve kısa adı "4'-Metilkalkon" (4'-Methylchalcone) olan "4'Metil-3-Fenilakrilofenon" $\left(\mathrm{C}_{16} \mathrm{H}_{14} \mathrm{O}\right)$ bileşiği sentezlendi. Çalışmamızda elde ettiğimiz bileşiğe benzer diğer çalışmalara literatürden ulaşmak mümkündür (4,4'-Dimetilkalkon [5-6-7] ve kalkon [8]). Elde ettiğimiz bileşiğin tek kristali elde edilip tek kristal $\mathrm{X}$-1şınları difraktometresinde verileri toplanarak bileşiğin kristal yapısı çözülmüştür. Çalışmamızda kullandığımı $\mathrm{C}_{16} \mathrm{H}_{14} \mathrm{O}$ bileşiğinin kimyasal diyagramı Şekil 1'de görülmektedir. Bileşik den elde edilen tek kristal, STOE IPDS II tek kristal X-1şını kırınımmetresine takılarak kırınım verileri toplandı. Bu veriler kullanılarak tek kristal yapı çözümü için yazılmış ShelxT bilgisayar programı ile kristalin yapısı çözüldü. Kristalin yapısının geometrik

\footnotetext{
*Sorumlu yazar: hinac@kku.edu.tr

Geliş Tarihi: 08.08.2018, Kabul Tarihi: 04.01.2019
} 
özellikleri Tablo 1'de, Ortep şekli Şekil 2'de ve bileşiğin moleküllerini bir arada tutarak kat1 yapıyı oluşturan hidrojen bağları verileri Tablo 2'de verildi.<smiles>CC(=O)c1ccc(C)cc1</smiles>

Şekil 1. Bileşiğin kimyasal diyagramı

\section{Materyal ve Metot}

\subsection{4'-Metil-3-Fenilakrilofenon Bileşiğinin Sentezi}

\% 95 etanol $(10 \mathrm{~mL})$ içindeki 4'-metilasetofenon (1.6 mL, $0.014 \mathrm{~mol})$ çözeltisine $(\% 10,10 \mathrm{~mL})$, benzaldehid (1.4 mL, $0.012 \mathrm{~mol}$ ) ilave edildi. Karışım 6 dakika oda sıcaklığında karıştıııldı ve sonra su $(10 \mathrm{~mL})$ ilave edildi. Karışım vakumlu filtrasyondan geçirildi. Sıcak etanolden yavaşça soğutularak renksiz (şeffaf) kristaller elde edildi. Bu kristallerden verisi toplanmak üzere örnek bir kristal seçildi.

\subsection{X-Işsını Kristalografisi}

STOE IPDS-II (Stoe Imaging Plate Difffraction System-II) difraktometresinde elde edilen veriler kullanılarak, X-RED isimli bilgisayar programı yardımı ile yapı çözümü için gerekli olan dosya oluşturuldu [9]. Kristalin yapısını çözmek için WinGX ([10]) paket programı içinde SHELXT ([11]) yapı çözüm programı kullanıldı. Yapı arıtımı için en-küçük kareler yöntemi SHELXL ([11]) programı kullanılarak gerçekleştirildi. Elde edilen sonuçların yayına hazırlanma aşamasında ORTEP-3 ([11]) çizim ve PLATON ([12]) hesaplama programları kullanıldı. Arıtım esnasında hidrojen atomları geometrik olarak (aromatik halka hidrojenleri için bağ uzunluğu $0,93 \AA$, metil hidrojenleri için bağ uzunluğu $0,96 \AA$ ) yerleştirilmiştir.

\section{Bulgular ve Tartışma}

Literatüre göre, Şekil 1'de kimyasal açık formülü görülen [4'-Metil-3-Fenilakrilofenon Bileşiği, $\left.\left(\mathrm{C}_{16} \mathrm{H}_{14} \mathrm{O}\right)\right]$ ve daha önce tek kristali elde edilip yapısı çözülmemiş olan bu yapı için elde edilen kristalin yapısı çözüldü. Bu çalışmada, Şekil 1'de kimyasal açık formülü görülen [4'-Metil-3-Fenilakrilofenon (C16H14O)] bileşiğin tek kristali elde edildi. Seçilen tek kristalin şiddet verileri X 1şını difraktometresi ile toplandı ve elde edilen veriler kristalografide kullanılan bilgisayar programları aracıllğı ile incelenerek kristalin yapısı çözüldü. Kristalin yapısının çözülmesi ile elde edilen Ortep şekli yapının çözümünden elde edilen atomik koordinatlar ve atomik yerdeğiştirme parametreleri kullanılarak oluşturulmuş ve Şekil 2'de verilmektedir. Şekildeki tüm yerleşim elipsoitleri \%30 olasıllkla çizilmiştir. Bileşiğin kristalografik verileri ve arıtım ayrıntıları Tablo 1'de verilmiştir. Bileşiğin tek kristalinin monoklinik $\mathrm{C} 2 / \mathrm{c}$ uzay grubunda kristallendiği, birim hücresinde sekiz molekül bulunduğu saptanmıştır. Kristal yapı herhangi bir çözücü molekül içermemektedir ve morfolojik yapısı prizma şeklinde ve renksiz (şeffaf)' dir. 


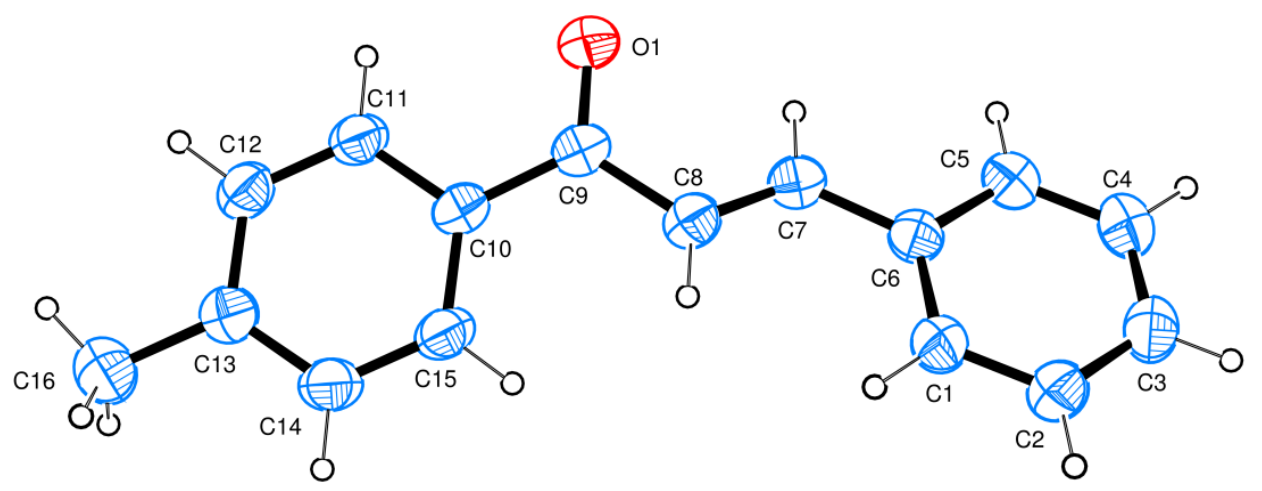

Şekil 2. Bileşiğin atomlarının numaralandırılmış çizimi

Moleküler yap1; bir metilvinilbenzen grubu ile bir benzen halkasının keton grubunun karbon atomları üzerinden birleşmesi ile meydana gelmiştir. Molekülün iki benzen halkası arasındaki açı değeri $26.995(8)^{\circ}$ olup molekülün düzlemsel yapıda olmadığını göstermektedir. Bu açı değeri literatürdeki benzer çalışmalarla uyum içerisindedir $\left(25.864(7)^{\circ}\right.$, [5] ve $28.324(9)^{\circ}$, [7]). Benzer olarak O1-C9$\mathrm{C} 8$ - C7 torsiyon açısı $-17.8(2)^{\circ}$ değerinde olup, Treadwell [7]'in yapmış olduğu çalışmada aynı torsiyon açısı $16,3(3)^{\circ}$ 'dir.

Tablo 1. Bileşiğin kristalografik verileri

\begin{tabular}{|c|c|}
\hline Kristal Verileri & \\
\hline Moleküler Formül & $\left(\mathrm{C}_{16} \mathrm{H}_{14} \mathrm{O}\right)$ \\
\hline Formül Ağırlığı (akb) & 222.27 \\
\hline Kristal sistemi, uzay grubu & Monoklinik, $C 2 / \mathrm{c}$ \\
\hline Sicaklık (K) & 296 \\
\hline $\mathrm{a}, \mathrm{b}, \mathrm{c}(\AA)$ & 14.9921(9), $9.8460(4), 17.5570(11)$ \\
\hline$\beta\left(^{\circ}\right)$ & $105.721(5)$ \\
\hline $\mathrm{V}\left(\AA^{3}\right)$ & $2494.7(2)$ \\
\hline $\mathrm{Z}$ & 8 \\
\hline $\mathrm{X}$-1şını tipi & Mo K $\alpha$ \\
\hline$\mu$ soğurma katsayısı $(\mathrm{mm}-1)$ & 0.07 \\
\hline Kristal boyutlar1 $(\mathrm{mm})$ & $0.50 \times 0.39 \times 0.25$ \\
\hline \multicolumn{2}{|l|}{ Veri Toplama } \\
\hline Difraktometre & STOE IPDS 2 \\
\hline Veri toplama sıcaklığı $(\mathrm{K})$ & 296 \\
\hline Soğurma Düzeltmesi & Integration \\
\hline$\theta \min , \theta \max$ & $2.41^{\circ}, 26.50^{\circ}$ \\
\hline $\begin{array}{l}\text { Ölçülen yansıma, bağımsız yansıma, gözlenen } \\
{[I>2 \sigma(I)] \text { yansıma }}\end{array}$ & $27623,2589,1776$ \\
\hline $\mathrm{T}_{\min }, \mathrm{T}_{\max }$ & $0.9686,0.9887$ \\
\hline $\mathrm{R}_{\text {int }}$ & 0.040 \\
\hline$(\sin \theta / \lambda)_{\max }\left(\AA^{-1}\right)$ & 0.628 \\
\hline \multicolumn{2}{|l|}{ Aritım } \\
\hline $\mathrm{R}\left[\mathrm{F}^{2}>2 \sigma\left(\mathrm{F}^{2}\right)\right], w \mathrm{R}\left(\mathrm{F}^{2}\right), \mathrm{S}$ & $0.043,0.111,1.06$ \\
\hline$\Delta \rho_{\max }, \Delta \rho_{\min }\left(\mathrm{e} \AA^{-3}\right)$ & $0.10,-0.12$ \\
\hline Hidrojen atomları & Geometriye göre yerleştirildi \\
\hline Yansıma sayıs1 & 2589 \\
\hline Parametre sayis1 & 155 \\
\hline
\end{tabular}


Bu bileşikteki moleküllerin kristal yapıyı oluşturabilmesi için molekülleri birbirine bağlayan istiflenmeyi oluşturan bir adet moleküller arası $\mathrm{C} 5-\mathrm{H} 5 \cdots \mathrm{O} 1 \mathrm{H}$-bağ geometrisi Tablo 2'de ve H-bağları ile oluşan sentrosimetrik dimerik yapı Şekil 3 'te gösterilmiştir.

Çalışmamızda Benzen halkalarının düzlemsellikten sapma değerinin $\mathrm{C} 1-\mathrm{C} 6$ benzen halkası için bulunan maksimum değer $\mathrm{C} 3$ atomunda $0.0067 \AA$, C10-C15 benzen halkası için bulunan maksimum değer C12 atomunda $0.0040 \AA$ olduğu tespit edildi. Benzen halkalarından; C1-C6 halkas1 için düzlemsellikten ortalama sapma $0.0048 \AA$ ve C10-C15 benzen halkası için $0.0024 \AA$ değerinde elde edildi. Ayrıca moleküler yapıya ait seçilen bazı bağ uzunlukları, bağ ve torsiyon açıları Tablo 3'te verilmiştir.

Tablo 2. Hidrojen bağ geometrisi $\left(\AA,^{\circ}\right)$

\begin{tabular}{|c|c|c|c|c|}
\hline $\mathbf{D}-\mathbf{H} \cdots \mathbf{A}$ & $\begin{array}{l}D- \\
\mathbf{H}\end{array}$ & $D \cdots A$ & $\mathbf{H} \cdots \mathbf{A}$ & $\mathbf{D}-\mathbf{H} \cdots \mathbf{A}$ \\
\hline $\mathrm{C} 5-\mathrm{H} 5 \cdots \mathrm{O} 1^{\mathrm{i}}$ & 0.93 & $3.37 .15(19)$ & 2.60 & 140.7 \\
\hline
\end{tabular}

Tablo 3. Seçilmiş bazı geometrik veriler $\left(\AA,{ }^{\circ}\right)$

\begin{tabular}{|l|l|l|l|}
\hline $\mathrm{C} 6-\mathrm{C} 7$ & $1.459(2)$ & $\mathrm{C} 9-\mathrm{C} 10$ & $1.484(2)$ \\
\hline $\mathrm{C} 9-\mathrm{O} 1$ & $1.2279(17)$ & $\mathrm{C} 8-\mathrm{C} 7$ & $1.321(2)$ \\
\hline $\mathrm{C} 9-\mathrm{C} 8$ & $1.474(2)$ & $\mathrm{C} 13-\mathrm{C} 16$ & $1.506(2)$ \\
\hline $\mathrm{O} 1-\mathrm{C} 9-\mathrm{C} 8$ & $119.63(14)$ & $\mathrm{C} 11-\mathrm{C} 10-\mathrm{C} 9$ & $119.39(13)$ \\
\hline $\mathrm{O} 1-\mathrm{C} 9-\mathrm{C} 10$ & $120.16(12)$ & $\mathrm{C} 15-\mathrm{C} 10-\mathrm{C} 9$ & $123.48(13)$ \\
\hline $\mathrm{O} 1-\mathrm{C} 9-\mathrm{C} 10-\mathrm{C} 11$ & $-4.0(2)$ & $\mathrm{C} 8-\mathrm{C} 9-\mathrm{C} 10-\mathrm{C} 11$ & $176.13(12)$ \\
\hline
\end{tabular}

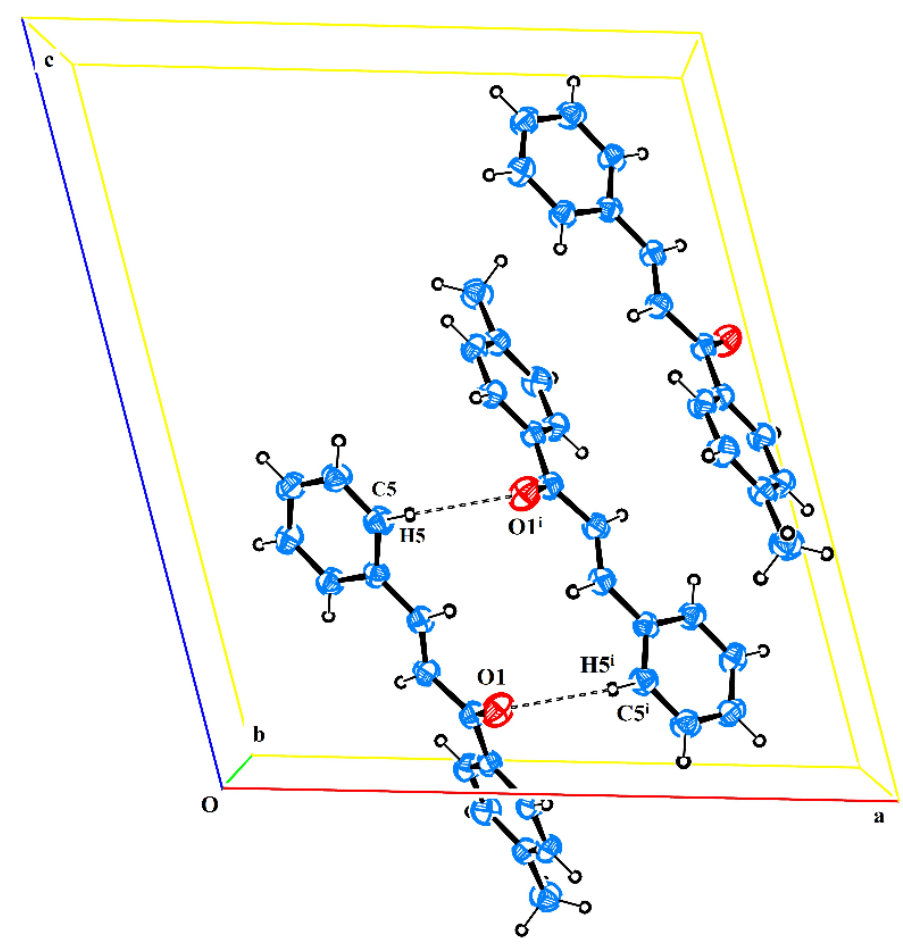

Şekil 3. Bileşiğin $\mathrm{C}-\mathrm{H} \cdots \mathrm{O}$ hidrojen bağlarıyla yaptığı paket yapının b ekseni boyunca çizimi. Kesikli çizgiler hidrojen bağlarını göstermektedir (simetri kodu i: -x, y, -z+3/2). 


\section{Sonuç ve Öneriler}

Bileşiğin moleküler yapısı bir metilvinilbenzen grubu ile bir benzen halkasının keton grubunun karbon atomları üzerinden birleşmesi ile meydana gelmiştir. Moleküler yapının düzlemselliği hem benzen halkaları ve hem de tüm molekül için ayrı ayrı araştırılmıştır. Benzen halkalarında düzlemsellikten en çok sapan atomların, C1-C6 benzen halkasında bulunan C3 atomu için maksimum değer $0.0067 \AA$ ve C10-C15 benzen halkasında bulunan C12 atomu için maksimum değer $0.0040 \AA$ olduğu gözlenmiştir. Benzen halkalarından; C1-C6 halkası için düzlemsellikten ortalama sapma $0.0048 \AA$ ve C10-C15 benzen halkası için düzlemsellikten ortalama sapma $0.0024 \AA$ değerinde elde edilmiştir. Oldukça küçük bu sapma değerleri ile her iki benzen halkasının da düzlemsel olduğu söylenebilir. Düzlemsel yapıda olmayan molekülün, $\mathrm{O} 1-\mathrm{C} 9-\mathrm{C} 8-\mathrm{C} 7$ torsiyon açıs1 $-17.8(2)^{\circ}$ dir. Treadwell [7]'in yapmış olduğu benzer bir çalışmada aynı torsiyon açısı $16,3(3)^{\circ}$ olarak verilmiştir. Moleküler yapıdaki iki benzen halkası da aynı düzlemde değildir. İki benzen halka düzlemi arasındaki dihedral açı $26.995(8)^{\circ}$ dir. Bu açı değeri literatürdeki benzer iki çalışmada $[7,8]$ surasıyla $25.864(7)^{\circ}, 28.324(9)^{\circ}$ 'lik değerleriyle, çalışmamızla uyum içindedir.

Kristal yapı C-H...O tipi zayıf hidrojen bağlarının yanı sıra van der Waals etkileşmeleri ile kararlı haldedir. Tablo 2'de geometrisi tanımlanan C-H...O etkileşmeleri ile moleküller sentrosimetrik dimerler oluşturacak biçimde bağlanarak kristal yapı içinde Şekil 3’te görüldüğü gibi istiflenmiştir. Ayrıca çalışmamızda elde edilen kristalografik verilerin literatürle uyum içinde olduğu görülmüştür [1315]. Bu bileşiğin kristal yapısının diğer verileri ekte verilen tablolardan görülebilir.

\section{Kaynaklar}

[1] Kapata P.C., Kapata N., Klinkenberg E., Grobusch M.P, Cobelens F. 2017. The prevalence of HIV among adults with pulmonary TB at a population level in Zambia, BMC infectious diseases, 17(1).

[2] Magiorakos A.P., Srinivasan A., Carey R.B., Carmeli Y., Falagas M.E., Giske C.E., Harbarth S., Hindler J.F., Kahlmeter G., Olsson B. 2012. Multidrugresistant, extensively drug-resistant and pandrug-resistant bacteria: an international expert proposal for interim standard definitions for acquired resistance, Clinical Microbiolgy and Infection, 18: 268-281.

[3] Tiwari R., Moraski G.C., Krchnak V., Miller P.A., Martinez M.C., Herrore E., Oliver A.G., Miller M.J. 2013. Thiolates chemically induce redox activation of BTZ043 and related potent nitroaromatic anti-tuberculosis agents. Journal of the American Chemical Society, 135: 3539-3549.

[4] Mahapatra D.K., Bharti S.K., Asati V. 2015. Chalcone scaffolds as antiinfective agents: Structural and molecular target perspectives. European Journal Medicinal Chemistry, 11: 496-524.

[5] Rabinovich D., Shakked Z. 1974. Optical Induction in Chiral Crystals. I. The Crystal and Molecular Structures of 4,4'-Dimethylchalcone, Acta Crystallographica Section B., B30: 2829-2834.

[6] Toda F., Tanaka K., Kato M. 1998. Stereoselective photodimerisation of chalcones in the molten state, J. Chem. Soc., Perkin Trans. 1, pp. 1315-1318.

[7] Treadwell E.M. 2006. 4-Methylchalcone. Acta Crystallographica Section E, E62: 05899-o5900.

[8] Rabinovich D. 1970. Topochemistry. Part XXX. Crystal and molecular structures of chalcone, Journal of the Chemical. Society. (B), pp. 11-16.

[9] Stoe C. 2002. X-Area(Version 1.18) ve X-Red32 (Version 1.04), Stoe ve Cie, Darmstadt, Germany.

[10] Farrugia L.J. 2012. WinGX and ORTEP for Windows: an update, Journal Applied Crystallography, 45: 849-854.

[11] Sheldrick G.M. 2015. SHELXT - Integrated space group and crystal structure determination, Acta Crystallographica Section A, A71, 3-8.

[12] Spek A.L. 2009. Structure validation in chemical crystallography, Acta Crystallographica Section D, D65: 148-155.

[13] Raghavendra S., Chidankumar C.S., Jayarama A., Dharmaprakash S.M. 2015. 1-[4(methylsulfanyl) phenyl]-3-(4-nitropshenyl) prop-2-en-1-one: A reverse saturable absorption based optical limiter, Materials Chemistry and Physics, 149: 487-494.

[14] Ekbote A., Patil P.S., Maidur S.R., Chia T.S., Quah C.K. 2017. Structure and nonlinear optical properties of (E)-1-(4-aminophenyl)-3-(3-chlorophenyl) prop-2-en-1-one: A promising new D-p- 
A-p-D type chalcone derivative crystal for nonlinear optical devices, Journal of Molecular Structure, 1129: 239-247.

[15] Zainuri D.A., Arshad S., Khalib N.C., Razak I.A., Pillai R., Sulaiman S.F., Hashim N.S., Ooi K.L., Armakovic S., Armakovic S.J., Panicker C.Y., Alsenoy C.V. 2017. Synthesis, XRD crystal structure, spectroscopic characterization (FT-IR, 1H and 13C NMR), DFT studies, chemical reactivity and bond dissociation energy studies using molecular dynamics simulations and evaluation of antimicrobial and antioxidant activities of a novel chalcone derivative, (E)-1-(4bromophenyl)-3-(4-iodophenyl)prop-2-en-1-one. Journal of Molecular Structure, 1128: 520-533.

\section{Ekler}

Ek A. Kesirsel atomik koordinatlar ve izotropik yerdeğiştirme parametreleri $\left(\AA^{2}\right)$

\begin{tabular}{l|l|l|l|l} 
& $x$ & $y$ & $z$ & $U_{\text {iso }} / U_{\text {eq }}$ \\
\hline C6 & $0.19464(9)$ & $0.32531(14)$ & $0.71966(8)$ & $0.0674(4)$ \\
\hline C9 & $0.10330(9)$ & $0.34044(15)$ & $0.90388(8)$ & $0.0711(4)$ \\
\hline C10 & $0.09865(8)$ & $0.25343(13)$ & $0.97171(8)$ & $0.0663(3)$ \\
\hline C8 & $0.15733(10)$ & $0.29643(15)$ & $0.84956(9)$ & $0.0751(4)$ \\
\hline H8 & 0.201641 & 0.228635 & 0.865607 & $0.090^{*}$ \\
\hline C15 & $0.13559(10)$ & $0.12317(15)$ & $0.98379(9)$ & $0.0804(4)$ \\
\hline H15 & 0.167341 & 0.088638 & 0.949301 & $0.096^{*}$ \\
\hline O1 & $0.06199(8)$ & $0.44943(11)$ & $0.89262(7)$ & $0.0936(4)$ \\
\hline C7 & $0.14436(10)$ & $0.35088(15)$ & $0.77865(9)$ & $0.0726(4)$ \\
\hline H7 & 0.096675 & 0.414160 & 0.764389 & $0.087^{*}$ \\
\hline C11 & $0.05224(10)$ & $0.30004(15)$ & $1.02482(9)$ & $0.0759(4)$ \\
\hline H11 & 0.026481 & 0.386615 & 1.018399 & $0.091^{*}$ \\
\hline C5 & $0.16803(10)$ & $0.39391(16)$ & $0.64791(9)$ & $0.0792(4)$ \\
\hline H5 & 0.117655 & 0.452743 & 0.637961 & $0.095^{*}$ \\
\hline C12 & $0.04349(10)$ & $0.22101(16)$ & $1.08677(9)$ & $0.0794(4)$ \\
\hline H12 & 0.012489 & 0.255566 & 1.121795 & $0.095^{*}$ \\
\hline C13 & $0.07980(10)$ & $0.09093(16)$ & $1.09829(9)$ & $0.0761(4)$ \\
\hline C1 & $0.26942(10)$ & $0.23740(15)$ & $0.73209(9)$ & $0.0778(4)$ \\
\hline H1 & 0.287970 & 0.189175 & 0.779296 & $0.093^{*}$ \\
\hline C3 & $0.28960(12)$ & $0.29079(18)$ & $0.60522(10)$ & $0.0889(5)$ \\
\hline H3 & 0.321997 & 0.280192 & 0.567316 & $0.107^{*}$ \\
\hline C4 & $0.21511(12)$ & $0.37616(18)$ & $0.59127(10)$ & $0.0899(5)$ \\
\hline H4 & 0.196119 & 0.422416 & 0.543408 & $0.108^{*}$ \\
\hline C14 & $0.12605(11)$ & $0.04453(16)$ & $1.04573(10)$ & $0.0857(4)$ \\
\hline H14 & 0.151521 & -0.042223 & 1.052251 & $0.103^{*}$ \\
\hline C16 & $0.06832(13)$ & $0.00394(19)$ & $1.16558(10)$ & $0.1017(5)$ \\
\hline H16A & 0.066802 & -0.090015 & 1.150669 & $0.153^{*}$ \\
\hline H16B & 0.011443 & 0.027242 & 1.177586 & $0.153^{*}$ \\
\hline H16C & 0.119403 & 0.019154 & 1.211370 & $0.153^{*}$ \\
\hline C2 & $0.31663(11)$ & $0.22045(17)$ & $0.67550(10)$ & $0.0869(4)$ \\
\hline H2 & 0.366850 & 0.161401 & 0.684775 & $0.104^{*}$ \\
& & & & \\
\hline
\end{tabular}


Ek B. Atomik yer değiştirme parametreleri $\left(\AA^{2}\right)$

\begin{tabular}{|c|c|c|c|c|c|c|}
\hline & $U^{11}$ & $U^{22}$ & $U^{33}$ & $U^{12}$ & $U^{13}$ & $U^{23}$ \\
\hline C6 & $0.0678(8)$ & $0.0633(8)$ & $0.0698(8)$ & $-0.0068(6)$ & $0.0161(6)$ & $-0.0041(6)$ \\
\hline C9 & $0.0673(8)$ & $0.0684(9)$ & $0.0768(9)$ & $0.0024(7)$ & $0.0184(6)$ & $-0.0099(7)$ \\
\hline C10 & $0.0615(7)$ & $0.0660(8)$ & $0.0698(8)$ & $0.0025(6)$ & $0.0152(6)$ & $-0.0121(7)$ \\
\hline C8 & $0.0730(8)$ & $0.0778(9)$ & $0.0741(9)$ & $0.0062(7)$ & $0.0192(7)$ & $-0.0053(7)$ \\
\hline C15 & $0.0848(9)$ & $0.0764(10)$ & $0.0834(10)$ & $0.0161(7)$ & $0.0286(8)$ & $-0.0087(8)$ \\
\hline O1 & $0.1091(8)$ & $0.0761(7)$ & $0.1067(8)$ & $0.0206(6)$ & $0.0481(7)$ & $0.0062(6)$ \\
\hline C7 & $0.0696(8)$ & $0.0682(8)$ & $0.0785(9)$ & $0.0017(6)$ & $0.0176(7)$ & $-0.0040(7)$ \\
\hline C11 & $0.0791(9)$ & $0.0696(9)$ & $0.0801(10)$ & $0.0132(7)$ & $0.0235(7)$ & $-0.0063(7)$ \\
\hline C5 & $0.0798(9)$ & $0.0759(10)$ & $0.0798(10)$ & $0.0001(7)$ & $0.0181(8)$ & $0.0075(8)$ \\
\hline C12 & $0.0791(9)$ & $0.0844(10)$ & $0.0787(10)$ & $0.0092(8)$ & $0.0280(7)$ & $-0.0069(8)$ \\
\hline C13 & $0.0720(8)$ & $0.0778(9)$ & $0.0744(9)$ & $-0.0002(7)$ & $0.0130(7)$ & $-0.0024(7)$ \\
\hline C1 & $0.0804(9)$ & $0.0778(9)$ & $0.0765(9)$ & $0.0044(7)$ & $0.0234(7)$ & $0.0063(7)$ \\
\hline C3 & $0.0956(11)$ & $0.0966(12)$ & $0.0818(11)$ & $-0.0115(9)$ & $0.0365(9)$ & $-0.0042(9)$ \\
\hline C4 & $0.0971(11)$ & $0.0944(12)$ & $0.0779(10)$ & $-0.0084(9)$ & $0.0235(9)$ & $0.0113(8)$ \\
\hline C14 & $0.0945(11)$ & $0.0697(10)$ & $0.0922(11)$ & $0.0155(8)$ & $0.0239(9)$ & $-0.0015(8)$ \\
\hline C16 & $0.1111(13)$ & $0.0988(12)$ & $0.0944(12)$ & $-0.0035(10)$ & $0.0267(10)$ & $0.0116(10)$ \\
\hline C2 & $0.0857(9)$ & $0.0864(10)$ & $0.0949(11)$ & $0.0062(8)$ & $0.0351(8)$ & $-0.0005(9)$ \\
\hline
\end{tabular}


Ek C. Geometrik parametreler $\left(\AA,{ }^{\circ}\right)$

\begin{tabular}{|c|c|c|c|}
\hline $\mathrm{C} 6-\mathrm{C} 1$ & $1.386(2)$ & $\mathrm{C} 15-\mathrm{C} 14$ & $1.374(2)$ \\
\hline $\mathrm{C} 6-\mathrm{C} 5$ & $1.3892(19)$ & $\mathrm{C} 11-\mathrm{C} 12$ & $1.372(2)$ \\
\hline $\mathrm{C} 6-\mathrm{C} 7$ & $1.459(2)$ & $\mathrm{C} 5-\mathrm{C} 4$ & $1.378(2)$ \\
\hline $\mathrm{C} 9-\mathrm{O} 1$ & $1.2279(17)$ & $\mathrm{C} 12-\mathrm{C} 13$ & $1.385(2)$ \\
\hline $\mathrm{C} 9-\mathrm{C} 8$ & $1.474(2)$ & $\mathrm{C} 13-\mathrm{C} 14$ & $1.374(2)$ \\
\hline $\mathrm{C} 9-\mathrm{C} 10$ & $1.484(2)$ & $\mathrm{C} 13-\mathrm{C} 16$ & $1.506(2)$ \\
\hline $\mathrm{C} 10-\mathrm{C} 11$ & $1.3842(18)$ & $\mathrm{C} 1-\mathrm{C} 2$ & $1.377(2)$ \\
\hline $\mathrm{C} 10-\mathrm{C} 15$ & $1.3904(19)$ & $\mathrm{C} 3-\mathrm{C} 4$ & $1.366(2)$ \\
\hline $\mathrm{C} 8-\mathrm{C} 7$ & $1.321(2)$ & $\mathrm{C} 3-\mathrm{C} 2$ & $1.377(2)$ \\
\hline $\mathrm{C} 1-\mathrm{C} 6-\mathrm{C} 5$ & $117.88(14)$ & $\mathrm{C} 12-\mathrm{C} 11-\mathrm{C} 10$ & $121.29(14)$ \\
\hline $\mathrm{C} 1-\mathrm{C} 6-\mathrm{C} 7$ & $123.21(14)$ & $\mathrm{C} 4-\mathrm{C} 5-\mathrm{C} 6$ & $121.03(15)$ \\
\hline $\mathrm{C} 5-\mathrm{C} 6-\mathrm{C} 7$ & $118.90(13)$ & $\mathrm{C} 11-\mathrm{C} 12-\mathrm{C} 13$ & $121.47(14)$ \\
\hline $\mathrm{O} 1-\mathrm{C} 9-\mathrm{C} 8$ & $119.63(14)$ & $\mathrm{C} 14-\mathrm{C} 13-\mathrm{C} 12$ & $117.34(14)$ \\
\hline $\mathrm{O} 1-\mathrm{C} 9-\mathrm{C} 10$ & $120.16(12)$ & $\mathrm{C} 14-\mathrm{C} 13-\mathrm{C} 16$ & $121.56(15)$ \\
\hline $\mathrm{C} 8-\mathrm{C} 9-\mathrm{C} 10$ & $120.21(13)$ & $\mathrm{C} 12-\mathrm{C} 13-\mathrm{C} 16$ & $121.10(15)$ \\
\hline $\mathrm{C} 11-\mathrm{C} 10-\mathrm{C} 15$ & $117.08(14)$ & $\mathrm{C} 2-\mathrm{C} 1-\mathrm{C} 6$ & $120.93(15)$ \\
\hline $\mathrm{C} 11-\mathrm{C} 10-\mathrm{C} 9$ & $119.39(13)$ & $\mathrm{C} 4-\mathrm{C} 3-\mathrm{C} 2$ & $119.88(16)$ \\
\hline $\mathrm{C} 15-\mathrm{C} 10-\mathrm{C} 9$ & $123.48(13)$ & $\mathrm{C} 3-\mathrm{C} 4-\mathrm{C} 5$ & $120.17(16)$ \\
\hline $\mathrm{C} 7-\mathrm{C} 8-\mathrm{C} 9$ & $121.62(14)$ & $\mathrm{C} 15-\mathrm{C} 14-\mathrm{C} 13$ & $121.61(15)$ \\
\hline $\mathrm{C} 14-\mathrm{C} 15-\mathrm{C} 10$ & $121.20(14)$ & $\mathrm{C} 3-\mathrm{C} 2-\mathrm{C} 1$ & $120.10(16)$ \\
\hline $\mathrm{C} 8-\mathrm{C} 7-\mathrm{C} 6$ & $128.82(14)$ & & \\
\hline $\mathrm{O} 1-\mathrm{C} 9-\mathrm{C} 10-\mathrm{C} 11$ & $-4.0(2)$ & $\mathrm{C} 7-\mathrm{C} 6-\mathrm{C} 5-\mathrm{C} 4$ & $178.08(14)$ \\
\hline $\mathrm{C} 8-\mathrm{C} 9-\mathrm{C} 10-\mathrm{C} 11$ & $176.13(12)$ & $\mathrm{C} 10-\mathrm{C} 11-\mathrm{C} 12-\mathrm{C} 13$ & $-0.7(2)$ \\
\hline $\mathrm{O} 1-\mathrm{C} 9-\mathrm{C} 10-\mathrm{C} 15$ & $173.30(14)$ & $\mathrm{C} 11-\mathrm{C} 12-\mathrm{C} 13-\mathrm{C} 14$ & $0.8(2)$ \\
\hline $\mathrm{C} 8-\mathrm{C} 9-\mathrm{C} 10-\mathrm{C} 15$ & $-6.6(2)$ & $\mathrm{C} 11-\mathrm{C} 12-\mathrm{C} 13-\mathrm{C} 16$ & $-178.85(14)$ \\
\hline $\mathrm{O} 1-\mathrm{C} 9-\mathrm{C} 8-\mathrm{C} 7$ & $-17.8(2)$ & $\mathrm{C} 5-\mathrm{C} 6-\mathrm{C} 1-\mathrm{C} 2$ & $1.0(2)$ \\
\hline $\mathrm{C} 10-\mathrm{C} 9-\mathrm{C} 8-\mathrm{C} 7$ & $162.09(13)$ & $\mathrm{C} 7-\mathrm{C} 6-\mathrm{C} 1-\mathrm{C} 2$ & $-177.67(14)$ \\
\hline $\mathrm{C} 11-\mathrm{C} 10-\mathrm{C} 15-\mathrm{C} 14$ & $0.1(2)$ & $\mathrm{C} 2-\mathrm{C} 3-\mathrm{C} 4-\mathrm{C} 5$ & $1.2(2)$ \\
\hline $\mathrm{C} 9-\mathrm{C} 10-\mathrm{C} 15-\mathrm{C} 14$ & $-177.23(14)$ & $\mathrm{C} 6-\mathrm{C} 5-\mathrm{C} 4-\mathrm{C} 3$ & $-0.4(2)$ \\
\hline $\mathrm{C} 9-\mathrm{C} 8-\mathrm{C} 7-\mathrm{C} 6$ & $176.22(13)$ & $\mathrm{C} 10-\mathrm{C} 15-\mathrm{C} 14-\mathrm{C} 13$ & $0.0(2)$ \\
\hline $\mathrm{C} 1-\mathrm{C} 6-\mathrm{C} 7-\mathrm{C} 8$ & $-2.1(2)$ & $\mathrm{C} 12-\mathrm{C} 13-\mathrm{C} 14-\mathrm{C} 15$ & $-0.4(2)$ \\
\hline $\mathrm{C} 5-\mathrm{C} 6-\mathrm{C} 7-\mathrm{C} 8$ & $179.27(14)$ & $\mathrm{C} 16-\mathrm{C} 13-\mathrm{C} 14-\mathrm{C} 15$ & $179.20(15)$ \\
\hline $\mathrm{C} 15-\mathrm{C} 10-\mathrm{C} 11-\mathrm{C} 12$ & $0.2(2)$ & $\mathrm{C} 4-\mathrm{C} 3-\mathrm{C} 2-\mathrm{C} 1$ & $-0.8(2)$ \\
\hline $\mathrm{C} 9-\mathrm{C} 10-\mathrm{C} 11-\mathrm{C} 12$ & $177.70(12)$ & $\mathrm{C} 6-\mathrm{C} 1-\mathrm{C} 2-\mathrm{C} 3$ & $-0.3(2)$ \\
\hline $\mathrm{C} 1-\mathrm{C} 6-\mathrm{C} 5-\mathrm{C} 4$ & $-0.7(2)$ & & \\
\hline
\end{tabular}

